Check for updates

Bogotá

Cite this as: $B M J 2022 ; 376: 0210$ http://dx.doi.org/10.1136/bmi.0210 Published: 25 January 2022

\section{Covid-19: CoronaVac immunity is strongest after boosting with a different vaccine}

\author{
Luke Taylor
}

A booster dose of a covid-19 jab other than CoronaVac significantly increased antibodies in those who had received two doses of the Chinese vaccine, a study funded by Brazil's Ministry of Health has found.

A booster dose of Pfizer Biontech's mRNA vaccine boosted antibody levels the most, followed by Oxford AstraZeneca, Janssen, and a third dose of CoronaVac. All booster vaccines were effective and safe, concluded the researchers at the University of Oxford, who co-led the study published in the Lancet. ${ }^{1}$

The findings will guide governments on how to boost their populations' immunity in the face of the more infectious omicron variant.

CoronaVac is manufactured by Chinese pharmaceutical Sinovac and is the world's most administered coronavirus vaccine, making up two of the 10 billion vaccine doses given globally. A preprint study has found CoronaVac to be less effective than mRNA vaccines and that its effectiveness drops further against omicron, ${ }^{2}$ so the findings are a boost for the many countries in Africa, Latin America, and South-east Asia that rely on the vaccine.

"The new data presented here show the extraordinary response to a third dose of coronavirus vaccines in a heterologous vaccine schedule," said Sue Ann Costa Clemens at the Oxford Vaccine Group and the study's lead author in Brazil.

The findings support Brazil's decision to use mRNA and viral vector vaccines as covid-19 boosters and "will also guide other low and middle income countries in setting up the most optimal and affordable booster programmes," she added.

To test the impact of the different boosters, 1240 participants who had received their second dose of CoronaVac six months prior in Sao Paulo and Salvador, Brazil, were given a randomised third dose of Oxford AstraZeneca, Pfizer BioNTech, Janssen, or CoronaVac.

Blood tests of the participants 28 days later showed that Pfizer increased their antibody levels 152 fold, Astrazeneca 90 fold, Janssen 77 fold, and CoronaVac 12-fold.

Neutralising antibodies, which bind to a virus in order to block infection, were detectable in $100 \%$ of those who received an Oxford AstraZeneca, Pfizer BioNTech, or Janssen booster, and in $83 \%$ of those who received a third dose of CoronaVac.

Only $20 \%$ of adults aged 18 to 60 and $9 \%$ of those over 60 had detectable levels of neutralising antibodies before the boosters.

The study did not assess levels of T cell immunity, which have been associated with protection from severe disease. The authors would not speculate on how the findings would translate to T cell protection, which will be examined in future studies.

The increase in antibody levels combined with the results of booster dose studies elsewhere-such the UK's CovBoost-suggest that boosters will offer strong protection against severe illness to those who have been vaccinated with CoronaVac.

Merryn Voysey, lead statistician at the Oxford Vaccine Group and the study's co-author, said, "Neutralising antibodies and binding antibodies have been shown to correlate with protection so high levels of antibody induced by these booster vaccines are likely to provide good protection against the original variants and also against severe disease in more recent variants.”

The findings follow other studies which found that mixing and matching different types of vaccines generally provokes a stronger immune response. "If you've got access and they're affordable, for vaccines, using a mixed schedule will get stronger responses," said Andrew Pollard, director of the Oxford Vaccine Group and the study's lead author.

Though studies show that mRNA vaccines would be optimal, they are not available for all countries, Pollard said. Only one in 10 people in low income countries have received a single dose of a covid-19 vaccine and distribution problems and costs continue to limit procurement options. ${ }^{3}$

"It's always about access to the product, the cost, and the type of immune response that you get. And we are still in a situation where many countries don't have access to the full range of vaccines," Pollard said. Along with the other co-authors he stressed that all the vaccines tested significantly boosted protection.

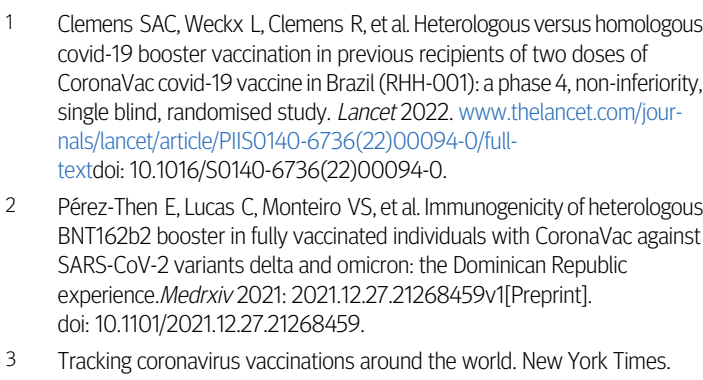

Clemens SAC, Weckx L, Clemens R, et al. Heterologous versus homologous covid-19 booster vaccination in previous recipients of two doses of CoronaVac covid-19 vaccine in Brazil (RHH-001): a phase 4, non-inferiority, single blind, randomised study. Lancet 2022. www.thelancet.com/journals/lancet/article/PIIS0140-6736(22)00094-0/fulltextdoi: 10.1016/S0140-6736(22)00094-0.

2 Pérez-Then E, Lucas C, Monteiro VS, et al. Immunogenicity of heterologous BNT162b2 booster in fully vaccinated individuals with CoronaVac against SARS-CoV-2 variants delta and omicron: the Dominican Republic experience.Medrxiv 2021: 2021.12.27.21268459v1[Preprint] doi: 10.1101/2021.12.27.21268459.

3 Tracking coronavirus vaccinations around the world. New York Times. www.nytimes.com/interactive/2021/world/covid-vaccinations-tracker.html.

This article is made freely available for personal use in accordance with BMJ's website terms and conditions for the duration of the covid-19 pandemic or until otherwise determined by BMJ. You may download and print the article for any lawful, non-commercial purpose (including text and data mining) provided that all copyright notices and trade marks are retained. 